\title{
Forearm bone mineral density and fracture incidence in postmenopausal women with osteoporosis: results from the ACTIVExtend phase 3 trial
}

\author{
N.B. Watts ${ }^{1}$ (D) R.K. Dore $^{2}$ (D) S. Baim ${ }^{3}$ (D) B. Mitlak ${ }^{4}$ (D) G. Hattersley ${ }^{4} \cdot$ Y. Wang $^{4}$ (D) T.D. Rozental ${ }^{5}$ (D) \\ M.S. LeBoff ${ }^{6}$ (iD
}

Received: 31 January 2020 / Accepted: 17 July 2020 / Published online: 15 September 2020

(C) The Author(s) 2020

\begin{abstract}
Summary Abaloparatide increased ultradistal radius bone mineral density (BMD) in the Abaloparatide Comparator Trial in Vertebral Endpoints (ACTIVE) trial. Over the subsequent 24 months in ACTIVExtend, ultradistal radius BMD gains were maintained with alendronate. Conversely, 1/3 radius BMD remained stable during ALN treatment in ACTIVExtend after decreasing during ACTIVE.

Introduction Abaloparatide (ABL) increased femoral neck, total hip, and lumbar spine bone mineral density (BMD) in postmenopausal women with osteoporosis and decreased the risk of vertebral and nonvertebral fractures in ACTIVE. Effects on fracture risk and BMD were maintained subsequently with alendronate (ALN) in ACTIVExtend. In a prespecified subanalysis of ACTIVE, ABL also increased BMD at the ultradistal radius. Our objective was to determine the efficacy of ABL followed by ALN vs placebo (PBO) followed by ALN on forearm BMD and fracture risk over 43 months in ACTIVExtend.

Methods Ultradistal and 1/3 radius BMD (ACTIVE baseline to month 43) were measured (ABL/ALN, $n=213 ; \mathrm{PBO} / \mathrm{ALN}, n=$ 233). Wrist fracture rates were estimated for the ACTIVExtend intent-to-treat population (ABL/ALN, $n=558 ; \mathrm{PBO} / \mathrm{ALN}, n=$ 581 ) by Kaplan-Meier (KM) method.

Results At cumulative month 25, mean increase from ACTIVE baseline in ultradistal radius BMD was 1.1\% (standard error, $0.49 \%)$ with ABL/ALN vs $-0.8 \%(0.43 \%)$ with PBO/ALN $(P<0.01)$. BMD increases with ABL were maintained with ALN through month 43 in ACTIVExtend. BMD decreases at the 1/3 radius in ACTIVE (similar with ABL and PBO) were maintained through 24 months of ALN treatment in ACTIVExtend. Wrist fractures over 43 months occurred in 15 women with ABL/ALN (KM estimate, $2.8 \%$ ) and 20 with PBO/ALN (KM estimate, 3.6\%) (HR=0.77, 95\% CI 0.39, 1.50; $P=$ not significant).

Conclusion Ultradistal radius BMD gains following treatment with ABL in ACTIVE were maintained over 24 months of ALN treatment in ACTIVExtend. Conversely, 1/3 radius BMD remained stable during ALN treatment in ACTIVExtend after decreasing during ACTIVE.

Trial registration ClinicalTrials.gov: NCT01657162 submitted July 31, 2012
\end{abstract}

Keywords Abaloparatide $\cdot$ Alendronate $\cdot$ Wrist fracture $\cdot$ Bone mineral density $\cdot$ Osteoporosis

B. Mitlak

bmitlak@radiuspharm.com

1 Mercy Health Osteoporosis and Bone Health Services, Cincinnati, OH, USA

2 Robin K. Dore, MD, Inc., Tustin, CA, USA

3 Rush University Medical Center, Chicago, IL, USA

4 Radius Health, Inc., 950 Winter Street, Waltham, MA 02451, USA

5 Beth Israel Deaconess Medical Center, Boston, MA, USA

6 Brigham and Women's Hospital, Boston, MA, USA

\section{Introduction}

Wrist fractures are the most common fracture of the upper extremity, and fractures of the distal radius account for approximately $18 \%$ of fractures in adults over 65 years of age $[1,2]$. Women with postmenopausal osteoporosis who have experienced a wrist fracture are $\sim 50 \%$ more likely to experience functional decline and are at increased risk of subsequent nonwrist fractures $[3,4]$.

Most fractures of the wrist occur at the ultradistal (UD) radius, which contains a high proportion of trabecular bone, so 
measurement of bone mineral density (BMD) at the UD radius may improve prediction of fracture compared with BMD measurement at the femoral neck alone [5-8]. However, the 1/3 radius, a region that is composed predominantly of denser cortical bone, is the forearm site most commonly reported by dualenergy x-ray absorptiometry (DXA) $[5,7,8]$.

Abaloparatide (ABL) is a selective activator of the parathyroid hormone receptor type 1 (PTHR1) signaling pathway, which results in direct stimulation of bone formation, improvement in bone microarchitecture, and enhanced measures of bone strength [9-11]. In the 18-month Abaloparatide Comparator Trial in Vertebral Endpoints (ACTIVE) phase 3 study, ABL significantly reduced the risk of vertebral, nonvertebral, and clinical fractures and increased BMD at the lumbar spine, total hip, and femoral neck compared with placebo (PBO) in postmenopausal women aged 49 to 86 years with osteoporosis [12]. ABL also significantly reduced the risk of major osteoporotic site fractures (collectively defined as clinical spine, hip, upper arm, and wrist) compared with patients treated with teriparatide (TPTD) and PBO [12]. In a prespecified subanalysis of ACTIVE, ABL significantly increased BMD at the UD radius vs $\mathrm{PBO}$ and TPTD, whereas BMD at the $1 / 3$ radius declined in all three treatment groups [13]. BMD effects were associated with a trend for numerically lower wrist fracture incidence for ABL $(n=7 / 824$ (Kaplan-Meier (KM) estimate, $1.0 \%)$ ) compared with TPTD $(n=17 / 818$ (KM estimate, $2.3 \%), P=0.052)$ and PBO $(n=15 / 821$ (KM estimate, 2.2\%), $P=0.11$ ) [13].

Women receiving ABL or PBO in ACTIVE were offered enrollment in the ACTIVExtend extension study in which both groups received 24 months of open-label alendronate (ALN) $70 \mathrm{mg} /$ week for a total of 43 months [14]. In ACTIVExtend, patients treated with ABL in ACTIVE who switched to ALN for 24 months had greater sustained reduction in fracture risk and increased BMD compared with PBO followed by ALN [14].

The objectives of this study, a prespecified subanalysis of ACTIVExtend, were to determine the efficacy of 18 months of ABL followed by 24 months of ALN (ABL/ALN) vs PBO followed by ALN (PBO/ALN) on BMD at the UD radius and $1 / 3$ radius and to assess the incidence of wrist fractures.

\section{Methods}

\section{Study design}

The study designs of ACTIVE and ACTIVExtend have been described in detail previously $[12,14]$. Briefly, ACTIVE enrolled 2463 postmenopausal women aged 49 to 86 years with osteoporosis. Postmenopausal women $\leq 65$ years of age were eligible if they had radiographic evidence of vertebral fracture at any time or a nonvertebral fracture within 5 years and had a BMD T-score of less than or equal to -2.5 but greater than -5.0 at the lumbar spine or femoral neck. Women older than 65 years who met fracture criteria but had a T-score of less than or equal to -2.0 and greater than -5.0 were also eligible. Women $>65$ years who did not meet the fracture criteria could enroll if their lumbar spine or femoral neck BMD T-score was less than or equal to -3.0 but greater than -5.0 .

ACTIVE participants were randomized 1:1:1 to 18 months of blinded treatment with ABL (Tymlos, Radius Health, Waltham, MA, USA) $80 \mu \mathrm{g}$ subcutaneously once daily or matching PBO or open-label treatment with TPTD $20 \mu \mathrm{g}$ once daily. Following completion of the ACTIVE study, there was an interval of approximately 1 month during which eligible participants were recruited and provided written consent to participate in ACTIVExtend. Participants eligible for ACTIVExtend included those who completed 18 months of blinded treatment with ABL or PBO in ACTIVE. Participants in the TPTD arm were not eligible to participate in ACTIVExtend. Study personnel reviewed patient diaries, and adherence to treatment was monitored at each clinic visit. Participants who were withdrawn from ACTIVE for any reason or who experienced a treatment-related serious adverse event during ACTIVE were also excluded.

All patients who participated in the extension study received open-label ALN monotherapy $70 \mathrm{mg}$ once per week for up to 24 months. Patients and investigators who participated in ACTIVE remained blinded to prior treatment assignment until all patients completed the first 6 months of ACTIVExtend.

ACTIVE and ACTIVExtend were approved by the ethics committee at all participating institutions and were conducted according to the recommendations of Good Clinical Practice and all other applicable local regulatory and ethical requirements and the Declaration of Helsinki (revised edition, Tokyo 2004). Written informed consent was obtained from all patients before any study-related procedures were performed.

\section{Study endpoints}

Changes from ACTIVE baseline BMD at the UD and 1/3 radius were assessed at 6 months (cumulative month 25) and 24 months (cumulative month 43). The forearm DXA subcohort included all ACTIVExtend patients who had radius ( $1 / 3$ radius and UD radius) BMD measurements at both ACTIVE baseline and at month 6 (cumulative month 25) in ACTIVExtend. In the ACTIVExtend intent-to-treat (ITT) study, clinical wrist fracture events (initially self-reported and subsequently verified from medical documents) included any wrist fractures for which the patient sought medical care, regardless of the level of trauma. Fractures were categorized as forearm, wrist, hand, and finger, with those categorized as wrist included in the analysis. 
Table 1 Baseline characteristics of the study participants in ACTIVExtend and forearm DXA subcohorts at ACTIVE baseline

\begin{tabular}{|c|c|c|c|c|}
\hline \multirow[b]{2}{*}{ Characteristic } & \multicolumn{2}{|l|}{ ABL/ALN } & \multicolumn{2}{|l|}{$\mathrm{PBO} / \mathrm{ALN}$} \\
\hline & $\begin{array}{l}\text { Overall } \\
(N=558)\end{array}$ & $\begin{array}{l}\text { Forearm DXA subcohort } \\
(N=213)\end{array}$ & $\begin{array}{l}\text { Overall } \\
(N=581)\end{array}$ & $\begin{array}{l}\text { Forearm DXA subcohort } \\
(N=233)\end{array}$ \\
\hline Age in years, mean (SD) & $68.6(6.5)$ & $68.7(6.1)$ & $68.5(6.3)$ & $68.0(6.4)$ \\
\hline \multicolumn{5}{|l|}{ Age group, $n(\%)$} \\
\hline$<65$ years & $106(19.0)$ & $39(18.3)$ & $114(19.6)$ & $55(23.6)$ \\
\hline 65 to $<75$ years & $351(62.9)$ & $139(65.3)$ & $370(63.7)$ & $141(60.5)$ \\
\hline$\geq 75$ years & $101(18.1)$ & $35(16.4)$ & $97(16.7)$ & $37(15.9)$ \\
\hline \multicolumn{5}{|l|}{ BMD T-score, mean (SD) } \\
\hline Lumbar spine & $-2.9(0.9)$ & $-2.8(0.8)$ & $-2.9(0.8)$ & $-2.8(0.9)$ \\
\hline Total hip & $-1.9(0.7)$ & $-2.0(0.7)$ & $-1.9(0.8)$ & $-2.0(0.8)$ \\
\hline Femoral neck & $-2.1(0.6)$ & $-2.3(0.6)$ & $-2.2(0.7)$ & $-2.2(0.7)$ \\
\hline $1 / 3$ radius & $-2.8(1.1)^{\mathrm{a}}$ & $-2.8(1.1)$ & $-2.8(1.2)^{\mathrm{a}}$ & $-2.8(1.2)$ \\
\hline Ultradistal radius & $-3.3(1.3)^{\mathrm{a}}$ & $-3.3(1.3)$ & $-3.4(1.3)^{\mathrm{a}}$ & $-3.4(1.3)$ \\
\hline Baseline prevalent vertebral fracture, $n / N(\%)^{\mathrm{b}}$ & $123 / 558(22.0)$ & $51 / 213(23.9)$ & $132 / 580(22.8)$ & $49 / 232(21.0)$ \\
\hline At least 1 prior nonvertebral fracture, $n / N(\%)^{\mathrm{c}}$ & $272 / 558(48.7)$ & $96 / 213(45.1)$ & $282 / 581(48.5)$ & $111 / 233(47.6)$ \\
\hline
\end{tabular}

ABL, abaloparatide; ALN, alendronate; BMD, bone mineral density; $\mathrm{PBO}$, placebo; SD, standard deviation

${ }^{\mathrm{a}} \mathrm{ABL} / \mathrm{ALN}(N=230) ; \mathrm{PBO} / \mathrm{ALN}(N=251)$

${ }^{\mathrm{b}}$ Evaluated by Bioclinica-Synarc

${ }^{\mathrm{c}}$ Excluded fractures of the spine, breastbone, kneecap, toes, fingers, skull, and facial bones

\section{Statistical analysis}

BMD at the UD and $1 / 3$ radius were measured by DXA (Lunar or Hologic) and were centrally analyzed (BioclinicaSynarc, Newark, CA, USA). Analyses of mean BMD differences between groups and change from baseline within groups should be interpreted in the context of limitations of DXA scanner precision for individual patients. Prior analyses of intra-individual variability have shown approximately $1 \%$ DXA scanner precision $[15,16]$. Percent changes in BMD from baseline were evaluated at each study visit, with missing data imputed based on last observation carried forward. Comparisons within treatment groups were performed using $t$ test. Comparisons among treatment groups were performed using analysis of covariance (ANCOVA) models. DXA scanner type and baseline BMD were included as covariates in ANCOVA models.

In the ACTIVExtend ITT analysis (558 ABL/ALN; 581 $\mathrm{PBO} / \mathrm{ALN}$ ), incident wrist fracture event rates were estimated by the Kaplan-Meier (K-M) method. The Cox
Fig. 1 Mean $( \pm \mathrm{SE})$ percent change in UD radius BMD from baseline through month 43 $* P<0.001$ vs $\mathrm{PBO} ; \dagger P<0.01$ vs $\mathrm{PBO} / \mathrm{ALN}$. ABL, abaloparatide; ALN, alendronate; BMD, bone mineral density; $\mathrm{PBO}$, placebo; $\mathrm{SE}$, standard error. $P$ value was based on the analysis of covariance model. Missing BMD data imputed using the method of last observation carried forward

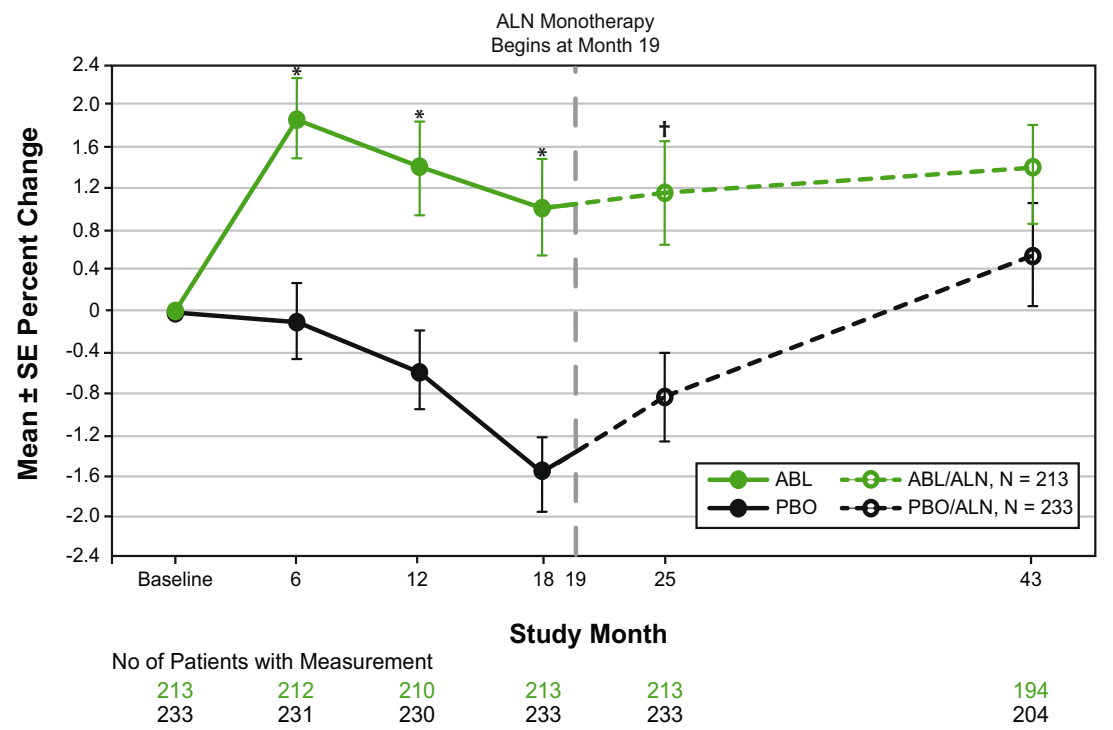


Fig. 2 Mean $( \pm$ SE) percent change in $1 / 3$ radius BMD from baseline through month 43 $\S P<0.05$ vs PBO. ABL, abaloparatide; ALN, alendronate; BMD, bone mineral density; $\mathrm{PBO}$, placebo; SE, standard error. $P$ value was based on the analysis of covariance model. Missing BMD data imputed using the method of last observation carried forward

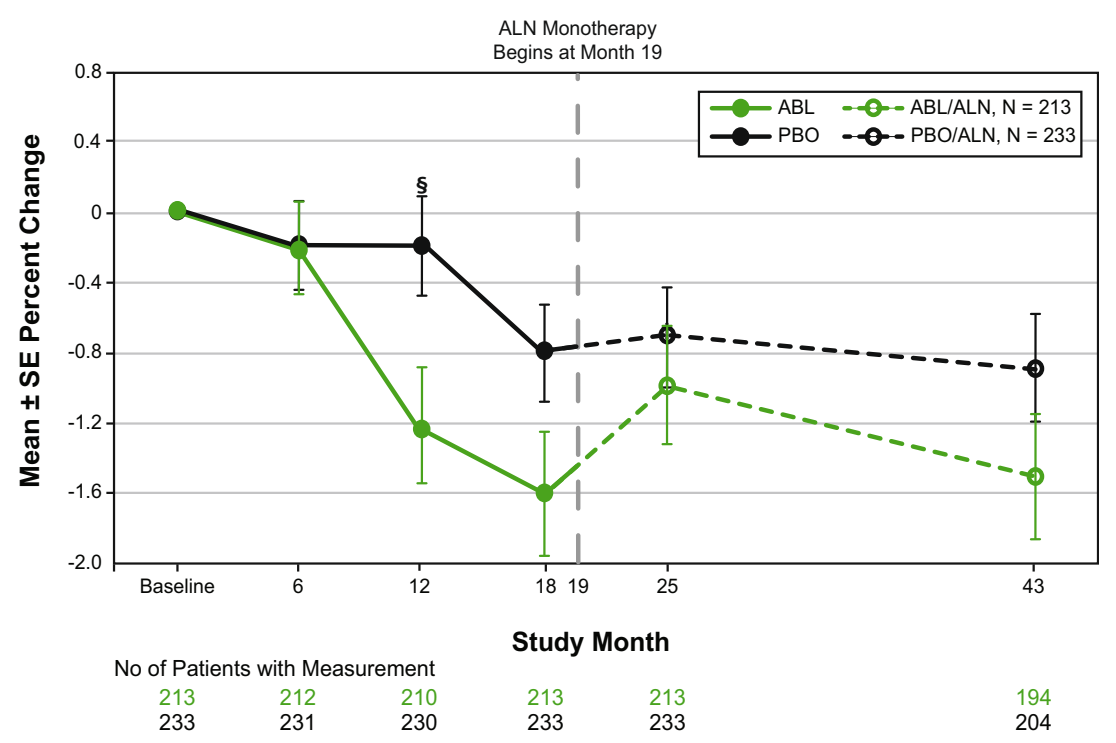

proportional hazard model was used to calculate the hazard ratio with $\mathrm{PBO} / \mathrm{ALN}$ as reference. $P$ values were from the log-rank test.

\section{Results}

\section{Characteristics of the study participants}

Demographic characteristics of study participants in ACTIVE and ACTIVExtend have been previously described $[12,14]$. A total of 446 postmenopausal women (ABL/ALN, $n=213$; $\mathrm{PBO} / \mathrm{ALN}, n=233$ ) were included in the forearm DXA study subcohort. Baseline characteristics (at ACTIVE baseline) were generally balanced between treatment groups for the overall ACTIVExtend population and in the forearm DXA ACTIVExtend study subcohort (Table 1). Mean (SD) baseline $1 / 3$ radius $\mathrm{BMD} \mathrm{T}$-score was $-2.8(1.1)$ and -2.8 (1.2) in the ABL/ALN and PBO/ALN groups, respectively. Mean (SD) baseline UD radius BMD T-score in the forearm DXA study was - 3.3 (1.3) and - 3.4 (1.3) in the ABL/ALN and $\mathrm{PBO} / \mathrm{ALN}$ groups, respectively.

\section{Bone mineral density}

Mean percent change from baseline in UD radius BMD increased with 18 months ABL treatment during ACTIVE [13]. BMD was maintained during 24 months treatment with ALN in the ABL/ALN group in ACTIVExtend through cumulative month 43 (Fig. 1). Conversely, UD radius BMD in the $\mathrm{PBO}$ group during ACTIVE decreased to below ACTIVE baseline [13]. BMD subsequently increased during 24 months treatment with ALN treatment in the PBO/ALN group in ACTIVExtend through cumulative month 43 (Fig. 1). Mean (standard error (SE)) percent change in UD radius BMD from ACTIVE baseline was $1.1(0.49)$ in the ABL/ALN group and $-0.8(0.43)$ in the PBO/ALN group after 6 months of ALN treatment (cumulative month 25 treatment difference, $2.1 \%$; $95 \%$ CI $0.83 \%, 3.32 \% ; P<0.01)$ and $1.3(0.49)$ in the ABL/ ALN group and $0.5(0.50)$ in the $\mathrm{PBO} / \mathrm{ALN}$ group at 24 months (cumulative month 43 treatment difference, $0.89 \%$; $95 \% \mathrm{CI}-0.46 \%, 2.24 \% ; P=0.20)$. Mean percent change in UD radius BMD from ACTIVE baseline in the ABL/ALN group was significantly different versus the $\mathrm{PBO} /$ ALN group at all time points except month 43.

Table 2 Time to event for clinical wrist fracture

\begin{tabular}{lll}
\hline & $\begin{array}{l}\text { ABL/ALN } \\
(N=558)\end{array}$ & $\begin{array}{l}\text { PBO/ALN } \\
(N=581)\end{array}$ \\
\hline Number of patients with event, $n(\%)$ & $15(2.7)$ & $20(3.4)$ \\
Kaplan-Meier estimated event rate at month 43, $\%$ & 2.8 \\
Hazard ratio (95\% CI) vs PBO/ALN & $0.77(0.39,1.50)$ \\
$P$ value vs PBO/ALN & 0.44 \\
\hline
\end{tabular}

ABL, abaloparatide; ALN, alendronate; CI, confidence interval; $\mathrm{PBO}$, placebo

${ }^{\text {a }}$ Cox proportional hazard model was used to calculate the hazard ratio with placebo/alendronate as reference

${ }^{\mathrm{b}} P$ values were from the log-rank test 
Fig. 3 Kaplan-Meier curve of time to first incident clinical wrist fracture from ACTIVE baseline through month 43. ABL, abaloparatide; ALN, alendronate; $\mathrm{HR}$, hazard ratio; NS, not significant; PBO, placebo

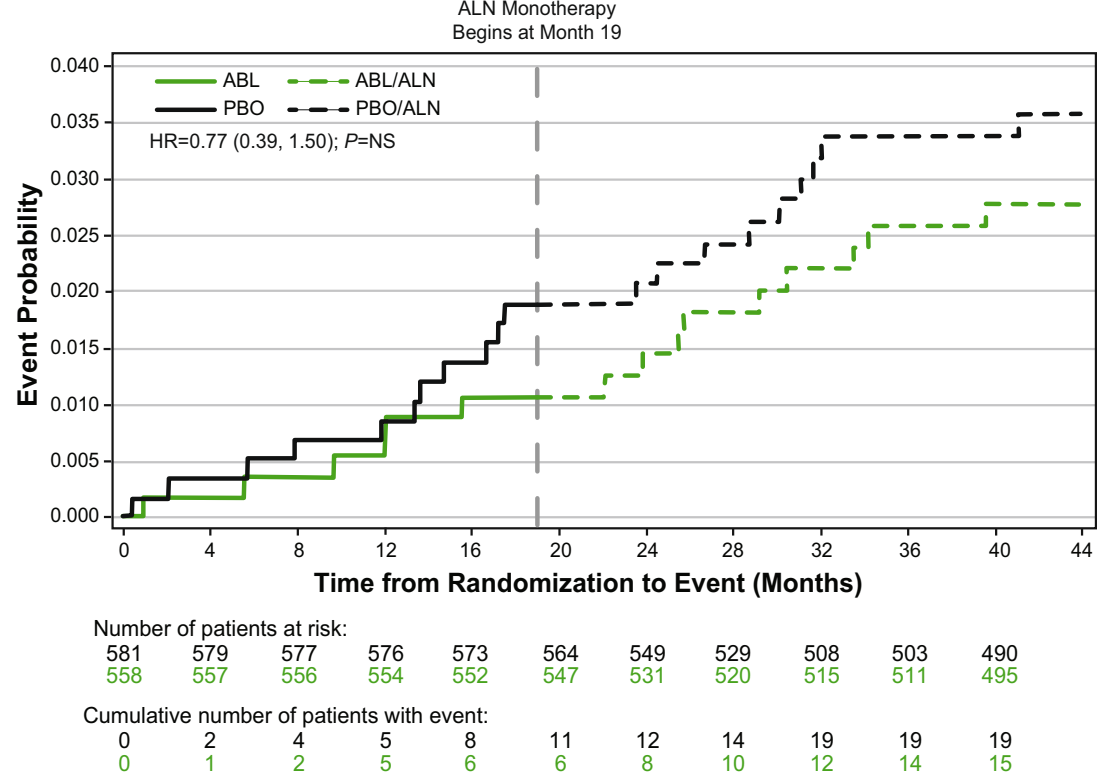

The values of $1 / 3$ radius $\mathrm{BMD}$ decreased through month 18 in both the ABL and PBO groups in ACTIVE [13]. Upon initiation of ALN treatment, the mean percent changes in 1/3 radius BMD from ACTIVExtend baseline were stable through month 24 (cumulative month 43) in the ABL/ALN and PBO/ALN groups (Fig. 2). At month 24 (cumulative month 43), mean (SE) percent change in $1 / 3$ radius BMD from ACTIVE baseline was - $1.5(0.35)$ for the ABL/ALN group and $-0.9(0.29)$ for the $\mathrm{PBO} /$ ALN group $(P=0.18)$. BMD values remained below ACTIVE baseline values at all time points through month 24 (cumulative month 43) in both treatment groups with no statistically significant differences between groups, except at month 12 (Fig. 2).

\section{Wrist fracture incidence}

The number of wrist fractures was low in both treatment groups (Table 2). In the ACTIVExtend population, the $\mathrm{K}-\mathrm{M}$-estimated event rate from ACTIVE baseline for clinical wrist fracture after the subsequent 24 months of ALN treatment (cumulative month 43) was lower in the $\mathrm{ABL} / \mathrm{ALN}$ group compared with the $\mathrm{PBO} / \mathrm{ALN}$ group ( $2.8 \%$ vs $3.6 \%$, respectively). The difference was not statistically significant (hazard ratio $(\mathrm{HR})=0.77 ; 95 \% \mathrm{CI}$ $0.39,1.50 ; P=0.44$ ) (Fig. 3). In the ACTIVE population, by month 18 , there was a separation between the ABL and PBO groups in time to wrist fracture that was maintained through 43 months with subsequent ALN treatment (2.7\% vs $3.9 \%$, respectively; $\mathrm{HR}=0.69 ; 95 \%$ CI 0.36 , $1.29 ; P=0.24)$; however, differences were not statistically significant.

\section{Discussion}

In the 18-month ACTIVE trial, BMD at the predominantly trabecular UD radius increased in women treated with ABL [13]. In this subanalysis of ACTIVExtend, results show that the increase in BMD at the UD radius is maintained in women in whom 18 months of ABL treatment was followed by treatment with ALN for 24 months. In contrast, BMD at the more cortical $1 / 3$ radius site decreased in both the $\mathrm{ABL}$ and $\mathrm{PBO}$ groups during ACTIVE [13], and BMD remained below baseline through 43 months in both groups in ACTIVExtend. Although the mean percent decrease in BMD at the 1/3 radius site in the ABL/ALN group was numerically greater than that observed in the PBO/ALN group, this difference was not significant at any time point in ACTIVExtend.

In ACTIVE, the increase in BMD at the UD radius in women treated with $\mathrm{ABL}$ compared with women receiving PBO was accompanied by numerically lower incidence of wrist fracture in the ABL group compared with the $\mathrm{PBO}$ group [13]. Our results are consistent with those reported in ACTIVE; although the number of wrist fractures was low in both groups, it was numerically higher in women treated with PBO followed by ALN compared with women treated with ABL followed by ALN. The K-M curve of time to first wrist fracture event showed a trend towards early separation between the ABL and PBO groups; this separation increased through the remainder of ACTIVE and was maintained with subsequent ALN treatment throughout ACTIVExtend, although there was no statistically significant difference between treatment groups. 
There is no consensus regarding the clinically significant threshold for percent change in BMD at the radius, and, overall, weaker associations have been reported between BMD changes and fracture risk at nonvertebral sites than the hip and vertebral sites [17]. Although BMD at the $1 / 3$ radial site is used more for clinical care, UD radius BMD has been shown to improve prediction of hip fractures when considered together with femoral neck BMD, likely owing to its combined trabecular and cortical bone structure [6]. The results of this study, together with the data reported in our earlier analysis of ACTIVE, [13] provide support for a potential role for assessment of BMD at the UD radius in helping to predict future fracture risk in postmenopausal women with osteoporosis.

A limitation of these analyses was the low number of patients who experienced a wrist fracture in both treatment arms of this study, limiting the statistical power to detect differences in wrist fracture incidence. In addition, DXA scanner precision at the $1 / 3$ and UD radius sites was not quantified. To better evaluate the clinical significance of BMD changes observed, a separate precision study is recommended for each skeletal site that is used to follow patients [15].

\section{Conclusions}

The BMD gains at the UD radius following treatment with ABL in ACTIVE were maintained over the subsequent 24 months of treatment with ALN in ACTIVExtend. Conversely, 1/3 radius BMD remained stable during ALN treatment in ACTIVExtend after decreasing during ACTIVE. Although not significant, the risk of wrist fractures was numerically lower with ABL/ALN compared with $\mathrm{PBO} /$ ALN. These results, together with previous ACTIVE analyses, suggest a potential correlation between UD wrist BMD and wrist fracture risk that warrants further evaluation in studies designed specifically to address this relationship.

Data sharing statement Data that underlie the results reported in a published article may be requested for further research 6 months after completion of the FDA or EMA regulatory review of a marketing application (if applicable) or 18 months after trial completion (whichever is latest). Radius will review requests individually to determine whether (i) the requests are legitimate and relevant and meet sound scientific research principles and (ii) are within the scope of the participants' informed consent. Prior to making data available, requestors will be required to agree in writing to certain obligations, including without limitation, compliance with applicable privacy and other laws and regulations. Proposals should be directed to info@ radiuspharm.com.

Funding Funding for this study (NCT01657162) was provided by Radius Health, Inc. Medical editorial support (Sarah Hummasti, PhD) and graphic services were provided by AOIC, LLC, and were funded by Radius Health, Inc. All listed authors meet the criteria for authorship set forth by the International Committee for Medical Journal Editors.

\section{Compliance with ethical standards}

Conflict of interest disclosure NB Watts is a speaker for Amgen and Radius Health, Inc., and a consultant for AbbVie, Amgen, and Sanofi. RK Dore is a speaker for Radius Health, Inc., and is a speaker, consultant, and has been involved in clinical trials for Amgen and Eli Lilly and Company. MS LeBoff receives grant/research support from the National Institute of Health/National Institute of Arthritis and Musculoskeletal and Skin Diseases and owns a company stock in Amgen. B Mitlak and Y Wang are employees of and own company stock in Radius Health, Inc. G Hattersley is a former employee of and consultant to Radius Health, Inc. S Baim declares that he has no conflicts of interest to disclose and TD Rozental is the Editor in Chief of the Journal of Hand Surgery Global Online.

Ethical approval All procedures performed in studies involving human participants were in accordance with the ethics committee at all participating institutions and were conducted according to the recommendations of Good Clinical Practice and all other applicable local regulatory and ethical requirements and the Declaration of Helsinki (revised edition, Tokyo 2004).

Open Access This article is licensed under a Creative Commons Attribution-NonCommercial 4.0 International License, which permits any non-commercial use, sharing, adaptation, distribution and reproduction in any medium or format, as long as you give appropriate credit to the original author(s) and the source, provide a link to the Creative Commons licence, and indicate if changes were made. The images or other third party material in this article are included in the article's Creative Commons licence, unless indicated otherwise in a credit line to the material. If material is not included in the article's Creative Commons licence and your intended use is not permitted by statutory regulation or exceeds the permitted use, you will need to obtain permission directly from the copyright holder. To view a copy of this licence, visit http:// creativecommons.org/licenses/by-nc/4.0/.

\section{References}

1. Baron JA, Karagas M, Barrett J, Kniffin W, Malenka D, Mayor M, Keller RB (1996) Basic epidemiology of fractures of the upper and lower limb among Americans over 65 years of age. Epidemiology 7:612-618

2. Wu JC, Strickland CD, Chambers JS (2019) Wrist fractures and osteoporosis. Ortho Clin North Am 50:211-221

3. Crandall CJ, Hovey KM, Cauley JA, Andrews CA, Curtis JR, Wactawski-Wende J, Wright NC, Li W, LeBoff MS (2015) Wrist fracture and risk of subsequent fracture: findings from the Women's Health Initiative study. J Bone Miner Res 30:2086-2095

4. Edwards BJ, Song J, Dunlop DD, Fink HA, Cauley JA (2010) Functional decline after incident wrist fractures-study of osteoporotic fractures: prospective cohort study. BMJ 341:c3324

5. Schlenker RA, VonSeggen WW (1976) The distribution of cortical and trabecular bone mass along the lengths of the radius and ulna and the implications for in vivo bone mass measurements. Calcif Tissue Res 20:41-52

6. Biver E, Durosier-Izart C, Chevalley T, van Rietbergen B, Rizzoli R, Ferrari S (2018) Evaluation of radius microstructure and areal bone mineral density improves fracture prediction in postmenopausal women. J Bone Miner Res 33:328-337

7. ISCD Official positions 2015. Adult and Pediatric (Accessed May 31, 2019) 
8. Goldfarb CA, Yin Y, Gilula LA, Fisher AJ, Boyer MI (2001) Wrist fractures: what the clinician wants to know. Radiology 219:11-28

9. Doyle N, Varela A, Haile S, Guldberg R, Kostenuik PJ, Ominsky MS, Smith SY, Hattersley G (2018) Abaloparatide, a novel PTH receptor agonist, increased bone mass and strength in ovariectomized cynomolgus monkeys by increasing bone formation without increasing bone resorption. Osteoporos Int 29:685-697

10. Hattersley G, Dean T, Corbin BA, Bahar H, Gardella TJ (2016) Binding selectivity of abaloparatide for PTH-type-1-receptor conformations and effects on downstream signaling. Endocrinology 157:141-149

11. Bahar H, Gallacher K, Downall J, Nelson CA, Shomali M, Hattersley G (2016) Six weeks of daily Abaloparatide treatment increased vertebral and femoral bone mineral density, microarchitecture and strength in ovariectomized osteopenic rats. Calcif Tissue Int 99:489-499

12. Miller PD, Hattersley G, Riis BJ, Williams GC, Lau E, Russo LA, Alexandersen P, Zerbini CAF, Hu MY, Harris AG, Fitzpatrick LA, Cosman F, Christiansen C, for the ACTIVE Study Investigators (2016) Effect of abaloparatide vs placebo on new vertebral fractures in postmenopausal women with osteoporosis: a randomized clinical trial. JAMA 316:722-733

13. Watts NB, Hattersley G, Fitzpatrick LA, Wang Y, Williams GC, Miller PD, Cosman F (2019) Abaloparatide effect on forearm bone mineral density and wrist fracture risk in postmenopausal women with osteoporosis. Osteoporos Int 30:1187-1194

14. Bone HG, Cosman F, Miller PD, Williams GC, Hattersley G, Russo LA, Alexandersen P, Zerbini CA, Hu MY, Harris AG, Fitzpatrick LA, Cosman F, Christiansen C, ACTIVE Study Investigators (2018) ACTIVExtend: 24 months of alendronate after 18 months of abaloparatide or placebo for postmenopausal osteoporosis. J Clin Endocrinol Metab 103:2949-2457

15. Bonnick SL, Johnston CC Jr, Kleerekoper M, Lindsay R, Miller P, Sherwood L, Siris E (2001) Importance of precision in bone density measurements. J Clin Densitom 4:105-110

16. Leder BZ, O'Dea LS, Zanchetta JR, Kumar P, Banks K, Banks K, McKay K, Lyttle CR, Hattersley G (2015) Effects of abaloparatide, a human parathyroid hormone-related peptide analog, on bone mineral density in postmenopausal women with osteoporosis. J Clin Endocrinol Metab 100:697-706

17. Bouxsein ML, Eastell R, Lui LY, Wu LA, de Papp AE, Grauer A, Marin F, Cauley JA, Bauer DC, Black DM (2019) Change in bone density and reduction in fracture risk: a meta-regression of published trials. J Bone Miner Res 34:632-642

Publisher's note Springer Nature remains neutral with regard to jurisdictional claims in published maps and institutional affiliations. 